\title{
Exploring Anxiety and Chain Reaction of Anxiety in COVID-19 Pandemic: A large population Web-based survey in Japan
}

\section{Jinman Zhuang}

Fujian Medical University

\section{Guoxi Cai}

Nagasaki University: Nagasaki Daigaku

\section{Yixiao Lu}

Nagasaki University: Nagasaki Daigaku

Yulan Lin

Fujian Medical University

\section{Liping Ping Wong}

University of Malaya: Universiti Malaya

\section{Zhijian Hu}

Fujian Medical University

\section{Taro Yamamoto}

Nagasaki University: Nagasaki Daigaku

\section{Kouichi Morita}

Nagasaki University: Nagasaki Daigaku

\section{Kiyoshi Aoyagi}

Fujian Medical University

Fei He ( $\square$ i.fei.he@fjmu.edu.cn )

Fujian Medical University

\section{Research Article}

Keywords: anxiety, chain reactions, COVID-19, STAI-6

Posted Date: April 19th, 2021

DOI: https://doi.org/10.21203/rs.3.rs-420996/v1

License: (1) (1) This work is licensed under a Creative Commons Attribution 4.0 International License.

Read Full License 


\section{Abstract}

Background: Anxiety plays an important role in psychology, explore the anxiety and its chain reactions can provide a good insight of the measures to address mental health problem caused by the COVID-19 (Coronavirus Disease 2019) epidemic.

Methods: A cross-sectional study using data collected via an online self-reported questionnaire was conduct on Japan during the COVID-19 pandemic. Using State-Trait Anxiety Inventory (STAI-6) to assess the level of anxiety, and explore the relationship of anxiety STAI-6 Score, sources of COVID-19 information, the influences of COVID-19, social anxiety symptoms, discrimination, and evaluation toward the government.

Results: 4,127 participants were included to the analysis. The level of anxiety is not equally distributed across the general population with different age, gender, educational level, occupation, income, presence of underlying disease and location $(P<0.05)$. The numbers of sources to get information about COVID-19 have a positive correlation with STAI-6 Score (Spearman'rho=0.176, $P<0.001)$. The influences caused by the pandemic are related to moderate-severe anxiety. Then the high level of anxiety would add to social anxiety (Spearman'rho $=0.04, P<0.05)$ and discrimination behaviors (Spearman'rho $=0.11, P<0.01$ ). Generalized additive model shows that high anxiety would lower the responders' evaluations of the preventive measures took by either national government or local government.

Conclusions: Our findings provide a statistical evidence for the chain reaction of anxiety, that anxiety reactions may vary in predictable ways. Further researches can focus on what types of strategic interventions can cut the chain response of anxiety, in order to address the mental health problems in a pandemic.

\section{Background}

Psychology's role in health care has significantly expanded in recent years and the level of anxiety is a key indicator of mental health(1), persistent moderate to severe anxiety symptoms have a negative consequence on psychological health. Excessive searches for health-related information are anxietyprovoking(2), and accumulation of individual anxiety may trigger a series of social anxiety symptom(3). It was noted that trust is beneficial for reducing anxiety $(4,5)$, in other words, anxiety may add distrust of relevant departments. What's more, at high level, anxiety may contribute to increased possibility of affected by a public health emergency(6) and influences of an pandemic may in turn to increase the level of anxiety. Thus, there may be a chain reaction of anxiety that haven't been proven.

As the epidemic outbreak of COVID-19, dramatic changes to normal life is emerging in many countries, general population were experienced psychological distress especially anxiety in the pandemic $(7,8)$. A recently published paper in Nature showed that anxiety among graduate students rose by $50 \%$ during pandemic compared with last year in the US(9). As for Japan, equally inevitably, the general public were suffered psychological distress in COVID-19 pandemic $(10,11)$. There were seven most affected 
prefectures (Saitama, Chiba, Tokyo, Kanagawa, Osaka, Hyogo, Fukuoka) before Japan state of nationwide emergency declaration these counties were defined as emergency area, while the rest were defined as non-emergency area. Assess anxiety and its chain responses of the COVID-19 epidemic can be an important way to develop and implement emotional regulation control strategies in Japan.

Previous studies suggested that anxiety may cause psychological and behavioral changes, but lack study performed to assess the chain reaction of anxiety, especially based on a period of infection disease pandemic. In this study, we use State-Trait Anxiety Inventory (STAI-6) to measure the level of anxiety, which is a self-report psychological test for adults designed to measure feelings of immediate anxiety (12). Our aim is to investigate the anxiety symptoms among the general public caused by COVID-19 in Japan, and in order to demonstrate chain reaction relate of anxiety in a pandemic, we explore the relationship of STAI-6 Score, sources of COVID-19 related information, the influences of COVID-19, social anxiety symptoms, discrimination, and evaluation of the government. In particular, we also discuss the differences between emergency area and non-emergency area.

\section{Methods}

\section{Study Participants and designs}

A cross-sectional web-based survey was adopted in this study, via an online research company, Cross Marketing Inc. Japan (https://www.cross-m.co.jp/en/) between 12 and 13 May, 2020 in Japan. The survey included a pool of approximately 2 million (the total population of Japan is 127 million) registered individuals. We use quota sampling to attain equal gender and age distributions representative of the Japanese population based on statistics from the Labor Force Survey, Ministry of Internal Affairs and Communications, Monetary incentive was given after accomplish the survey. In the end of investigation, a total of 4134 participants responded to the questionnaire, after data cleaning (the respondents with logically inconsistent answers were excluded), 4127 complete responses were analyzed in this study.

\section{Questionnaire design}

This study consisted of questions that 1) demographic characteristics (included age gender, education, occupation, income, location, with or without underlying disease); 2) Individual anxiety; 3) Sources of information to receive COVID-19 related news; 4 ) influences to individuals or their family of COVID-19 epidemic; 5) social anxiety related to COVID-19; 6) discriminatory related to COVID-19; 7) evaluate score of the preventive activities by the national and local government.

To assess individuals' anxiety level related to the COVID-19 epidemic, the State-Trait Anxiety Inventory (STAI-6) was used(Marteau \& Bekker, 1992). This scale contains six items and four choices $(1=$ not at all, 2 = somewhat, $3=$ moderately, 4 = very much) that reflect the common symptoms of anxiety experienced by an individual. The scores on the three positively worded items were reverse-coded, the man who has higher score means he has a higher level of individual anxiety. The total summed scores were prorated (multiplied by 20/6) to obtain scores that were comparable with those from the full 20 -item STAI, the man who scores of 44 or above were defined as moderate to severe symptoms $(13,14)$. 
The question about sources of information regarding COVID-19 is a multiple choice that includes TV, radio, government authorities, internet or social network service (SNS), newspaper, family or friends and others. We used self-report method to explore the influences caused by COVID-19, participants can choose more than one answer in this item (includes the impact on individuals and family).

The section of social anxiety consisted of 5 items that included fear, avoidance, keep secret, embarrassment and stigma. And the acts of discrimination contained the feelings of don't want to contact anyone who related to COVID-19 even though they were no contagious. There are four answer options for the section of social anxiety and discriminations in the original questionnaire, we simplified the answers as "Yes" (Strongly agree and Agree) and "No" (Strongly disagree and Disagree).

Scoring system were adopted to evaluate the preventive measures took by the national government and local government. The scores range from 0 to 100 , with higher scores indicating the higher evaluation on government.

The questionnaire was adopted from previous study (15).

\section{Data analysis}

Means, frequencies and standard deviations (SDs) were used to quantify the data. We use Student's ttest, F-test and rank-sum test to identify differences of STAI-6 Score in different demographic background. The sources of COVID-19 related information, social anxiety and discrimination related to COVID-19 are correlated to STAI-6 Score using Spearman correlation test. We use generalized additive model to analysis the interaction of the evaluate score of the preventive activities by the national and local government and their correlation with STAI-6 Score. Chi-square $\left(\chi^{2}\right)$ were used to identify the relationship of individual anxiety and the influences to individuals or their family.

Data were analyzed using STATA/MP version 15.0 (StataCorp, College Station, TX, United States) and R software version 4.0.2 for Windows 10. Excel 2019 were used to drawing pictures.

\section{Results}

\section{Demographic characteristics and STAI-6 Score}

There are 2,187 respondents from emergency area and 1,940 respondents from non-emergency area. The differences in mean of STAI-6 Score were statistically significant in age, gender, highest educational level, occupation, income, underlying disease and location $(P<0.05)$. Participants (male, junior college or vocational school and above, informal occupation, annually household income is $200-500$ (10,000 JPY), have underlying disease) from non-emergency area had higher mean of STAl-6 Score than emergency area. The detailed characteristics and STAI-6 Score of the participants are presented in Table 1.

\section{Sources of information and STAl-6 Score}


The differences of sources to receive COVID-19 related news in different location were showed in Fig. 1. The number of participants from non-emergency area to get information relate to COVID-19 in Newspaper $(P<0.01)$ and family or friends $(P<0.05)$ were larger than the respondents from emergency area. The numbers of sources to get information about COVID-19 have a positive correlation with STAI-6 Score (Spearman'rho $=0.176, P<0.001 ;$ Fig. 2). Different location also received the same result (Emergency area: Spearman'rho $=0.166, P<0.001$; Non-emergency area: Spearman'rho $=0.186, P<$ $0.001)$.

\section{Influences of COVID-19 and individual anxiety}

Figure 3 shows the differences in COVID-19 epidemic influences between different location and the relationship of influences and individual anxiety. Participants with influences like social connection become weak, intake of alcohol increased, smoking increased, sleep quality is affected and gained weight are more from emergency area $(P<0.05)$. The respondents under the influence of increase in family quarrel and conflicts, social connection become week, sleep quality is affected, increase of stress, become nervous toward people around and people around me become nervous and anxiety are more likely to indicating moderate to severe individual anxiety symptoms $(P<0.05)$.

\section{Social anxiety, discriminatory and STAI-6 Score}

The summary of social anxiety and discriminatory related to COVID-19 in different location were shown in Table 2. In all participants, there are positive correlation between numbers of social anxiety symptom, numbers of discriminatory behavior, and STAI-6 score. In other words, increased numbers of social anxiety symptom and discriminatory behavior were associated with decreased STAI-6 Scores. Participant from non-emergency area had similar result. While in emergency area, there can't be find any correlation between STAI-6 Score and numbers of social anxiety symptom. (Figure 4)

\section{Evaluate score of government and STAI-6 Score}

The generalized additive model include location and evaluate score of the preventive activities by the national and local government as independent variables, STAI-6 Score as dependent variable.

Participants' evaluate score of the preventive activities by the national and local government had reciprocal action. The respondents who had higher STAI-6 Score, their evaluate score of the preventive activities by the national and local government were lower $(P<0.05)$. Participants from emergency area had lower STAI-6 Score (Intercept Estimate $=-0.298, P<0.05$ ). The visualization of interaction effect is shown in Fig. 5.

\section{Discussion}

Our study provides an insight of chain reaction related to individual anxiety during the COVID-19 epidemic. The number of accesses to information regarding COVID-19 will increase anxiety and influences under COVID-19 will add the possibility of moderate to severe anxiety symptoms, then the increased level of anxiety will lead to social anxiety symptoms, discriminatory behaviors, and distrust in 
governments. In addition, we suspect that high level of anxiety also may increase the possibility of being affected by COVID-19 pandemic.

To data, numerous studies had explored the anxiety of general public, health workers, teachers, students, or patients with certain diseases during the on-going COVID-19 pandemic. These studies can be broadly divided into three categories. The first one has been studied most, major in research the risk factor (including sociodemographic factors, online social media information, knowledge of COVID-19) of the anxiety(16-19). Second type of researches were concentrated in the effects after intervention on the anxiety caused by COVID-19 pandemic(20-22), interventions mainly consist of relaxation techniques like breath relaxation training and progressive muscle relaxation. The instructions for these interventions were audio-recorded and then put online, then the researchers assessed the anxiety status before and after the intervention. The third kind focus on what anxiety influenced our life, behaviors change caused by anxiety were common among the general public during COVID-19 epidemic(23). And these studies of anxiety related to work efficiency mainly conducted on a certain type of worker(11), while the investigate of patients in hospitals were to explore the change of somatic function under anxiety(24). To our knowledge, there is short of studies to explore anxiety related chain reaction which can be well known by people but never proved by real data during the COVID-19 pandemic. Our finding can provide a clear serial response of anxiety and offer informative implications to address the mental problems in a pandemic.

In our investigation, anxiety is not equally distributed across the general population with different age, gender, educational level, occupation, income, history of underlying disease and location. The result was in accordance with a recent national investigation in Japan(25).

In the pandemic, People were instructed to stay at home during the pandemic, in particular the emergency area, the sources of COVID-19 related information play a central role in understanding the status of epidemic. Our study found that the highest two concentrations of information resources are TV and internet or Social Networking Services (SNS). The responders from non-emergency area get information from newspaper and family or friends were higher the emergency area. Those findings are in line with current precautionary measures took by Japan.

Indeed, acquire information from a variety of different sources can add fears about the information and generate the anxiety(26). In this study, the numbers of resources to getting COVID-19 information and anxiety level were found strongly positive correlation in both emergency and non-emergency area, it confirmed a conjecture that getting numerous messages about an epidemic will increase the level of anxiety. A report conducted in Russia found that excessive media consumption about COVID-19 is related to the anxiety(27), it confirms that get a wealth of relevant information can increase anxiety related COVID-19.

Concerning the influences of individuals or their families by COVID-19 pandemic, we find that the responders from emergency area are more likely to affected by the epidemic. In view of this, we speculate that, in the period of people had to stay at home, family quarrel and conflicts may increase and their social connect became weak. Due to the diminution of social intercourse, they may become nervous 
towards others and thought that others were nervous too. Then they felt stress increasing and sleeping quality were affected. And that the influences regarding COVID-19 are related to anxiety symptoms, our present study prompts that the people who vulnerable to the effects of a pandemic is more likely to indicating moderate to severe individual anxiety. As early as the period of Severe Acute Respiratory Syndrome (SARS) pandemic, researchers were found that the persons who under the influences of SARS (such as feels of more stress(28) and more fear(29) were more prone to anxiety. Now the COVID-19 epidemic, the same reaction was found in our study, proved the feasibility of our research.

Currently, the social anxiety and discrimination behaviors were high-profile in this pandemic(30,31). Our finding indicated that social anxiety symptoms and discrimination behaviors may be the result of high level of individual anxiety. What's more, those three were pairs interact each other, it means that social anxiety also can increase the discrimination behaviors. A previous study on immigrants suggested that perceived discrimination would increase social anxiety symptoms(32), while our study first discuss the individual discrimination behaviors and social anxiety symptoms, the result shows that the person who discriminate against COVID-19 positive person or relevant health workers also suffered more social anxiety symptoms.

There we use generalized additive model to explore the relationship between the trust in governments (national and local government) and anxiety, we also consider the reciprocal action of evaluation on governments which means the effect of one variable on the result will change when the other variable changes. Findings indicated that with the effect of different regions, participants with lower evaluation of the government's preventive measures would have a higher level of anxiety. Besides, there is a reciprocal action between the evaluation in two levels of government. These finding are consistent with other studies showing that, the trust and anxiety can be predicted each other and have a negative relationship $(33,34)$.

Our investigation showed that impact under COVID-19 outbreak can increases the likelihood of anxiety. We suspect, the person with higher level of anxiety also can increase the influences of COVID-19 pandemic. Previous reports have pointed out that individuals with high anxiety were easier to affected by disease or public health emergency $(35,36)$. It showed that anxiety add the possibility to effected from a pandemic, which confirmed our conjecture.

Study limitations warrant consideration. First, our online investigation may lack the participants who do not use the network, this biases clearly limit the generality of our results. When it comes to the design of our questionnaire, our survey was designed for the public, the questions for specific population groups were not involved (such as the people who had been confirmed as positive infected). In addition, selfreport questionnaires have a bias about recall difficulties and may influenced by social desirability biases. Moreover, our cross-sectional study is difficult to infer causal relationship. Thus, studies employing multiple follow-up assessments are needed to determine anxiety and its related chain reaction. Despite limitations, our internet-based sampling strategy is the ability to quickly deploy a survey and thereby track responses in near real-time and carried out under large sample size and varies of 
demographic backgrounds. Besides, we used the standardized questionnaire (STAl-6) to diagnose anxiety, it supported us to give a reasonable conclusion. The findings can provide an insight to the anxiety and related chain responses in an epidemic.

\section{Conclusions}

This current study is the first to provide a probable evidence in chain reaction of anxiety that increased accesses to information of COVID-19 and affected by the outbreak would add anxiety, then high level of anxiety increased the social anxiety, discrimination behaviors, distrust on governments and may boost the possibility of stay under the influences of COVID-19 in turn. Our findings may help the psychologists have a better knowledge of anxiety and serve as a critical step in developing strategic plan to address later dimensions of anxiety in a pandemic. Furthermore, it may be generalized to other diseases or traumatic events.

\section{List Of Abbreviations}

COVID-19: Coronavirus Disease 2019

STAI-6: State-Trait Anxiety Inventory

\section{Declarations}

\section{Ethics approval and consent to participate}

This study was conducted after the approve of the Research Ethics Committee of Nagasaki Prefectural Institute of Environment and Public Health (No. 2020-6-1). Participants were informed that their participation was voluntary and they had the option to withdraw from the survey at any time before their participation. We use an online consent which was approved by the ethics committee to obtain the informed consent of participants. The data are completely anonymous.

\section{Consent for publication}

All of the authors are consent of publication.

\section{Availability of data and materials}

All data for this article are available from the corresponding author.

\section{Conflicts of interest}

The author reports no conflicts of interest in this work.

\section{Funding}


This work was supported by the Nagasaki Prefectural Research Project [grant number 2020FY-NIEP], JSPS KAKENHI [grant number JP19K24679] and Fujian Program for Outstanding Young Researchers in University awarded by Education Department of Fujian [grant number 2017B019]

\section{Author contributions}

Jinman Zhuang: Formal analysis, Writing - Original draft.

Guoxi Cai: Study design, Data curation, Investigation, Methodology, Writing - Review \& Editing, Funding acquisition.

Yixiao Lu: Investigation, Methodology, Writing - Review \& Editing.

Yulan Lin: Investigation, Methodology, Writing - Review \& Editing.

Li Ping Wong: Methodology, Writing - Review \& Editing, Supervision.

Zhijian Hu: Methodology, Writing - Review \& Editing, Supervision.

Taro Yamamoto: Methodology, Writing - Review \& Editing, Supervision.

Kouichi Morita: Methodology, Writing - Review \& Editing, Supervision, Funding acquisition.

Kiyoshi Aoyagi: Methodology, Writing - Review \& Editing, Supervision.

Fei He: Study design, Methodology, Formal analysis, Writing - Reviewing \& Editing, Supervision, Funding acquisition.

\section{Acknowledgements}

The authors are grateful to all participants for their contribution to the study.

\section{Authors' information}

Department of Epidemiology and Health Statistics, Fujian Provincial Key Laboratory of Environment Factors and Cancer, School of Public Health, Fujian Medical University, Fuzhou 350122, Fujian Province, China (Jinman Zhuang, Guoxi Cai, Yulan Lin, Zhijian Hu, Kiyoshi Aoyagi, Fei He)

Department of Public Health, Nagasaki Prefectural Institute of Environment and Public Health, Nagasaki, 856-0026, Japan (Guoxi Cai)

Department of International Health, Institute of Tropical Medicine (NEKKEN), Nagasaki University, Nagasaki, 852-8523, Japan (Guoxi Cai, Taro Yamamoto)

Department of Public Health, Nagasaki University Graduate School of Biomedical Sciences, Nagasaki, 852-8523, Japan (Yixiao Lu) 
Centre for Epidemiology and Evidence-Based Practice, Department of Social and Preventive Medicine, Faculty of Medicine, University of Malaya 50603, Kuala Lumpur, Malaysia (Ping Wong)

Department of Virology, Institute of Tropical Medicine (NEKKEN), Nagasaki University, Nagasaki 8528523, Japan (Kouichi Morita)

\section{References}

1. Horenstein A, Heimberg R. Anxiety disorders and healthcare utilization: A systematic review. Clinical psychology review. 2020;81:101894.

2. McMullan R, Berle D, Arnáez S, Starcevic V. The relationships between health anxiety, online health information seeking, and cyberchondria: Systematic review and meta-analysis. Journal of affective disorders. 2019;245:270-8.

3. Ng-Cordell E, Hanley M, Kelly A, Riby D. Anxiety in Williams Syndrome: The Role of Social Behaviour, Executive Functions and Change Over Time. Journal of autism and developmental disorders. 2018;48(3):796-808.

4. Jhang F. Changes in trust and trait anxiety: The mediating role of changes in self-rated health among older adults in Taiwan. Geriatrics \& gerontology international. 2020.

5. Flugelman M, Jaffe R, Luria G, Yagil D. Trust in the referring physician reduces anxiety in an integrated community-to-hospital care system. Israel journal of health policy research. 2020;9(1):7.

6. Tull M, Barbano A, Scamaldo K, Richmond J, Edmonds K, Rose J, et al. The prospective influence of COVID-19 affective risk assessments and intolerance of uncertainty on later dimensions of health anxiety. Journal of anxiety disorders. 2020;75:102290.

7. Wang H, Xia Q, Xiong Z, Li Z, Xiang W, Yuan Y, et al. The psychological distress and coping styles in the early stages of the 2019 coronavirus disease (COVID-19) epidemic in the general mainland Chinese population: A web-based survey. PloS one. 2020;15(5):e0233410.

8. Liu X, Luo W, Li Y, Li C, Hong Z, Chen H, et al. Psychological status and behavior changes of the public during the COVID-19 epidemic in China. Infectious diseases of poverty. 2020;9(1):58.

9. Woolston C. Signs of depression and anxiety soar among US graduate students during pandemic. Nature. 2020.

10. Shigemura J, Ursano R, Morganstein J, Kurosawa M, Benedek D. Public responses to the novel 2019 coronavirus (2019-nCoV) in Japan: Mental health consequences and target populations. Psychiatry and clinical neurosciences. 2020;74(4):281-2.

11. Sasaki N, Kuroda R, Tsuno K, Kawakami N. Workplace responses to COVID-19 associated with mental health and work performance of employees in Japan. Journal of occupational health. 2020;62(1):e12134.

12. Sydeman S. State-Trait Anxiety Inventory. 
13. Knight R, Waal-Manning H, Spears G. Some norms and reliability data for the State-Trait Anxiety Inventory and the Zung Self-Rating Depression scale. The British journal of clinical psychology. 1983:245-9.

14. Marteau T, Bekker $H$. The development of a six-item short-form of the state scale of the Spielberger State-Trait Anxiety Inventory (STAI). The British journal of clinical psychology. 1992;31(3):301-6.

15. Guoxi Cai YL, Yixiao Lu, Fei He, Kouichi Morita, Taro Yamamoto, Kiyoshi Aoyagi, Toshitsugu Taguri, Zhijian Hu, Haridah Alias, Mahmoud Danaee, Li Ping Wong. . Behavioural responses and anxiety symptoms during to the coronavirus disease 2019 (COVID-19) pandemic in Japan: A large scale cross-sectional study. Journal of Psychiatric Research 2020 (submitted).

16. Jungmann S, Witthöft M. Health anxiety, cyberchondria, and coping in the current COVID-19 pandemic: Which factors are related to coronavirus anxiety? Journal of anxiety disorders. 2020;73:102239.

17. Yang X, Zhang Y, Li S, Chen X. Risk factors for anxiety of otolaryngology healthcare workers in Hubei province fighting coronavirus disease 2019 (COVID-19). Social psychiatry and psychiatric epidemiology. 2020.

18. Jo W, Lee J, Park J, Kim Y. Online Information Exchange and Anxiety Spread in the Early Stage of the Novel Coronavirus (COVID-19) Outbreak in South Korea: Structural Topic Model and Network Analysis. Journal of medical Internet research. 2020;22(6):e19455.

19. Liu C, Yang Y, Zhang X, Xu X, Dou Q, Zhang W, et al. The prevalence and influencing factors in anxiety in medical workers fighting COVID-19 in China: a cross-sectional survey. Epidemiology and infection. 2020;148:e98.

20. Wei N, Huang B, Lu S, Hu J, Zhou X, Hu C, et al. Efficacy of internet-based integrated intervention on depression and anxiety symptoms in patients with COVID-19. Journal of Zhejiang University Science B. $2020 ; 21(5): 400-4$.

21. Chen S. An online solution focused brief therapy for adolescent anxiety during the novel coronavirus disease (COVID-19) pandemic: a structured summary of a study protocol for a randomised controlled trial. Trials. 2020;21(1):402.

22. Liu K, Chen Y, Wu D, Lin R, Wang Z, Pan L. Effects of progressive muscle relaxation on anxiety and sleep quality in patients with COVID-19. Complementary therapies in clinical practice. 2020;39:101132.

23. Stanton R, To Q, Khalesi S, Williams S, Alley S, Thwaite T, et al. Depression, Anxiety and Stress during COVID-19: Associations with Changes in Physical Activity, Sleep, Tobacco and Alcohol Use in Australian Adults. International journal of environmental research and public health. 2020;17(11).

24. Speth M, Singer-Cornelius T, Oberle M, Gengler I, Brockmeier S, Sedaghat A. Mood, Anxiety and Olfactory Dysfunction in COVID-19: Evidence of Central Nervous System Involvement? The Laryngoscope. 2020.

25. Qian K, Yahara T. Mentality and behavior in COVID-19 emergency status in Japan: Influence of personality, morality and ideology. PloS one. 2020;15(7):e0235883. 
26. Jones J, Salathé $M$. Early assessment of anxiety and behavioral response to novel swine-origin influenza A(H1N1). PloS one. 2009;4(12):e8032.

27. Nekliudov N, Blyuss O, Cheung K, Petrou L, Genuneit J, Sushentsev N, et al. Excessive media consumption about COVID-19 is associated with increased state anxiety: Outcomes of a large online survey in Russia. Journal of medical Internet research. 2020.

28. McAlonan G, Lee A, Cheung V, Cheung C, Tsang K, Sham P, et al. Immediate and sustained psychological impact of an emerging infectious disease outbreak on health care workers. Canadian journal of psychiatry Revue canadienne de psychiatrie. 2007;52(4):241-7.

29. Leung G, Ho L, Chan S, Ho S, Bacon-Shone J, Choy R, et al. Longitudinal assessment of community psychobehavioral responses during and after the 2003 outbreak of severe acute respiratory syndrome in Hong Kong. Clinical infectious diseases : an official publication of the Infectious Diseases Society of America. 2005;40(12):1713-20.

30. Choi S, Hong J, Kim Y, Park H. Predicting Psychological Distress Amid the COVID-19 Pandemic by Machine Learning: Discrimination and Coping Mechanisms of Korean Immigrants in the U.S. International journal of environmental research and public health. 2020;17(17).

31. Zheng L, Miao M, Lim J, Li M, Nie S, Zhang X. Is Lockdown Bad for Social Anxiety in COVID-19 Regions?: A National Study in The SOR Perspective. International journal of environmental research and public health. 2020;17(12).

32. Fang $\mathrm{K}$, Friedlander M, Pieterse A. Contributions of acculturation, enculturation, discrimination, and personality traits to social anxiety among Chinese immigrants: A context-specific assessment. Cultural diversity \& ethnic minority psychology. 2016;22(1):58-68.

33. Zwingmann J, Baile W, Schmier J, Bernhard J, Keller M. Effects of patient-centered communication on anxiety, negative affect, and trust in the physician in delivering a cancer diagnosis: A randomized, experimental study. Cancer. 2017;123(16):3167-75.

34. Quintana P, Nolet K, Baus O, Bouchard S. The Effect of Exposure to Fear-Related Body Odorants on Anxiety and Interpersonal Trust Toward a Virtual Character. Chemical senses. 2019;44(9):683-92.

35. Yang L, Yu Z, Xu Y, Liu W, Liu L, Mao H. Mental status of patients with chronic insomnia in China during COVID-19 epidemic. The International journal of social psychiatry. 2020;66(8):821-6.

36. Asmundson G, Taylor S. How health anxiety influences responses to viral outbreaks like COVID-19: What all decision-makers, health authorities, and health care professionals need to know. Journal of anxiety disorders. 2020;71:102211.

\section{Tables}

Due to technical limitations the Tables are available as a download in the Supplementary Files.

\section{Figures}




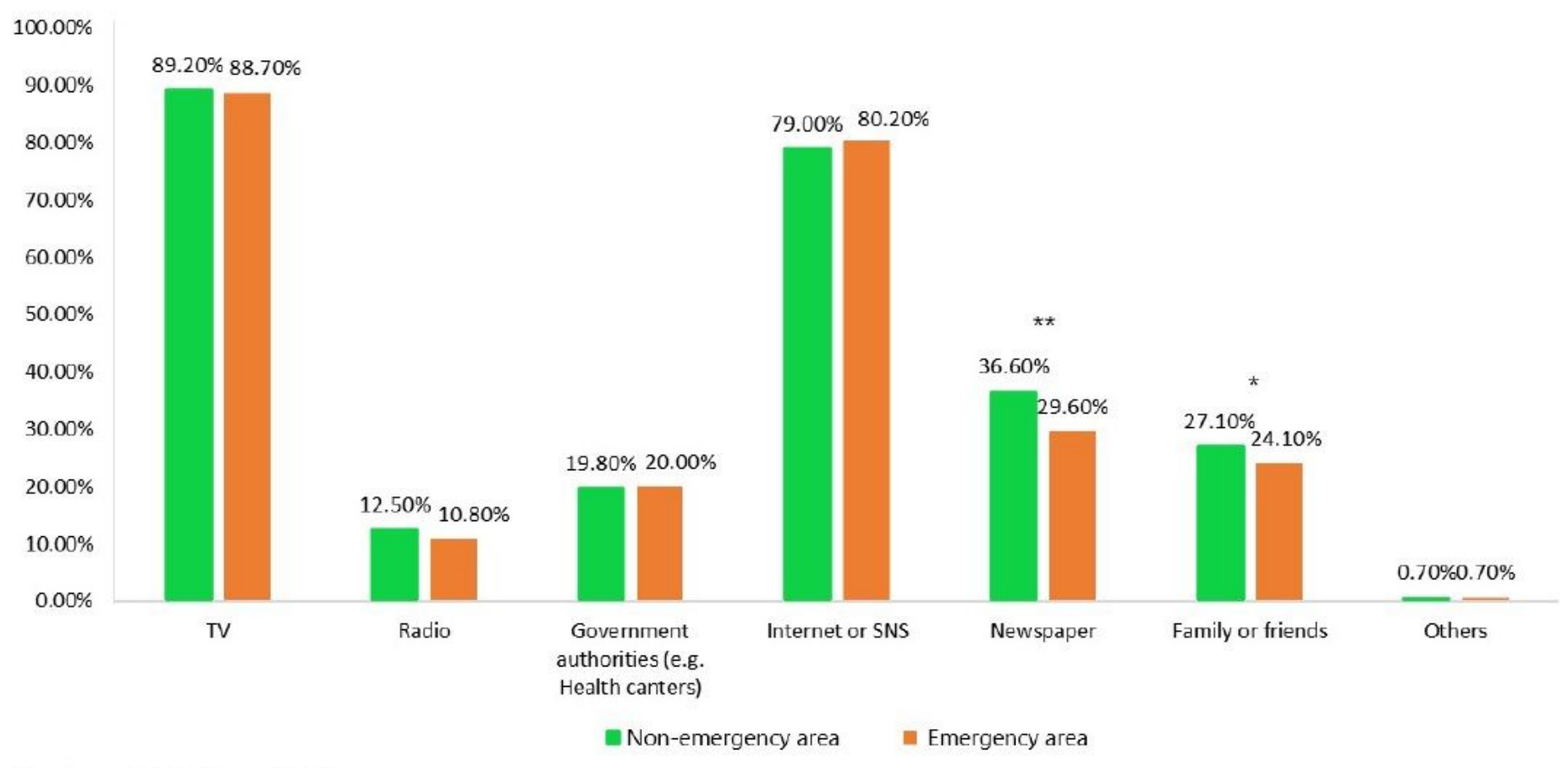

Note: *means $P<0.05$; **means $P<0.01$

\section{Figure 1}

Sources of information for participants in different locations to receive COVID-19 related news

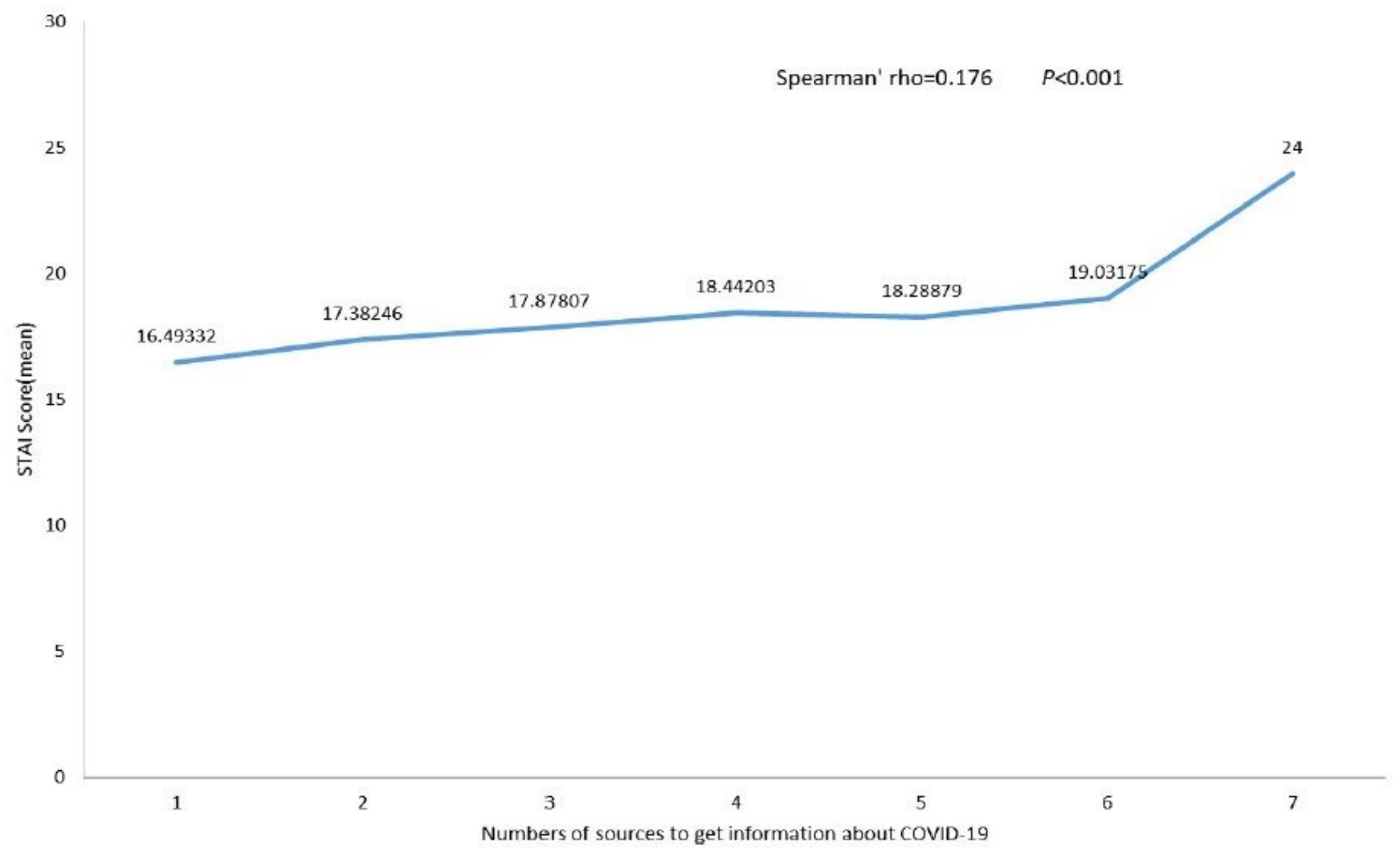

Figure 2 
The relationship of STAI-6 Score and numbers of sources to get information about COVID-19

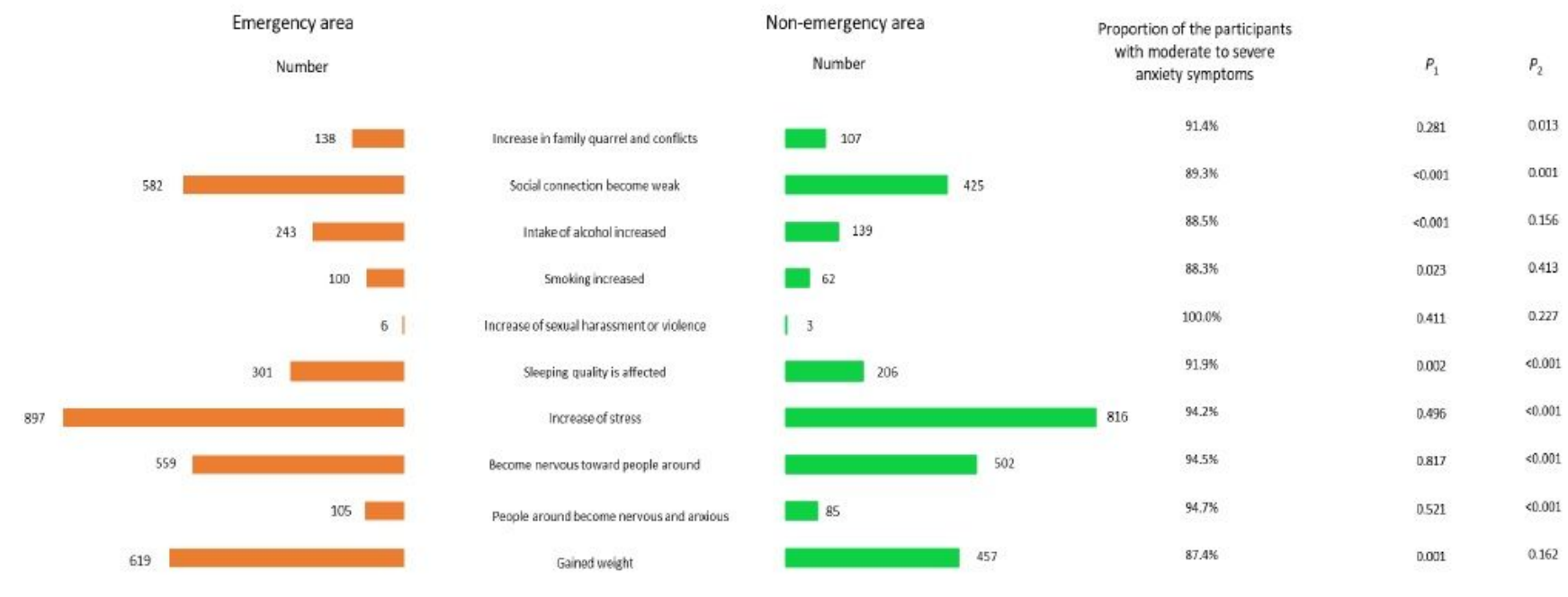

Note: P1 means the significance of the differences in COVID-19 epidemic influences between different location; P2 means the significance of the differences in moderate to severe anxiety symptoms incidence about the participants with or with COVID-19 epidemic influences

\section{Figure 3}

The situation of COVID-19 epidemic influence the participants or their family and the relation of moderate to severe anxiety symptoms

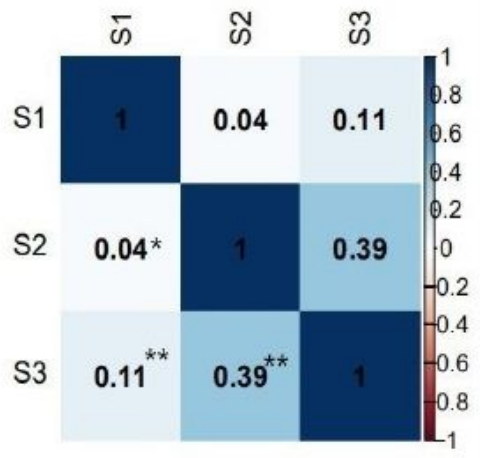

(a) All participants

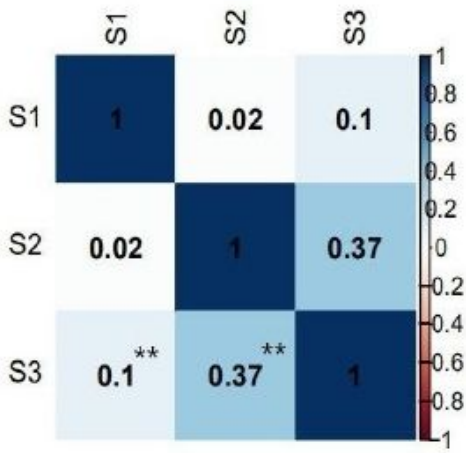

(b) Participants from emergency area

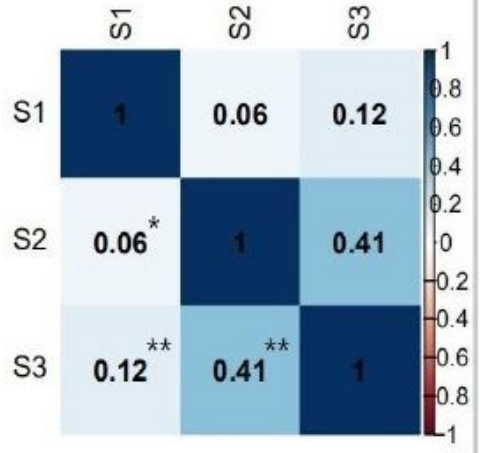

(c) Participants from non-emergency area

Note: S1=STAI-6 Score; S2=Numbers of social anxiety symptom; S3=Numbers of discriminatory behavior related to COVID-19.

*means $P<0.05 ;{ }^{* *}$ means $P<0.01$

\section{Figure 4}

The relationship between STAI-6 Score and numbers of social anxiety symptom and discriminatory behavior related to COVID-19 


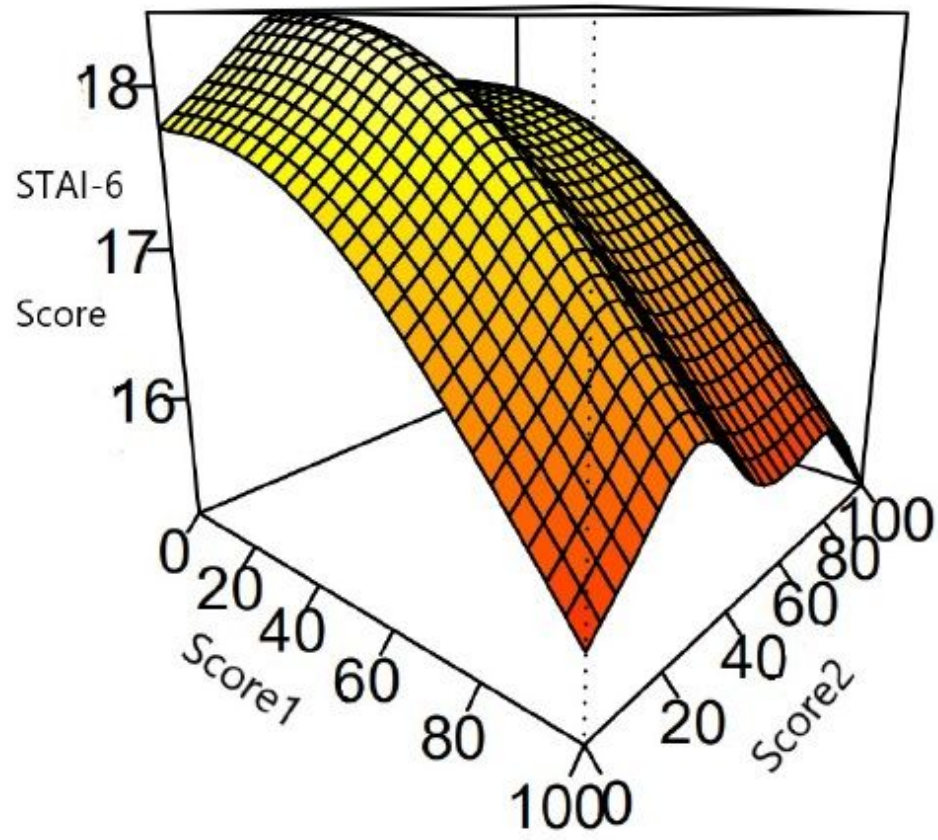

Note: Score1=Evaluate score of the preventive activities by the national government; Score2=Evaluate score of the preventive activities by the local government.

Figure 5

The interaction of evaluate score of governments affecting the STAI-6 Score

\section{Supplementary Files}

This is a list of supplementary files associated with this preprint. Click to download.

- Tables.pdf 